\title{
Blockchain, Tokenization, and Implications for Financial Services Practitioners
}

\author{
Sean Stein Smith \\ Assistant Professor, Lehman College \\ City University of New York, US \\ Tel: 551-206-7995Ｅ-mail: drseansteinsmith@gmail.com
}

Received: November 30, 2018 Accepted: December 19, 2018 Published: January 3, 2019

doi:10.5296/ijafr.v9i1.14164

URL: https://doi.org/10.5296/ijafr.v9i1.14164

\begin{abstract}
Blockchain technology represents perhaps the most powerful and transformative technology to enter the financial services landscape in the last several decades according to both practitioner commentary and academic research. While much has been written and analyzed about blockchain terminology and definitions, however, more recent applications and developments potentially hold more both challenges and opportunities for the financial services industry. This research examines the area of tokens, tokenization of physical assets, and the implications this continued shift and development has for both practitioners and academics. Lastly, and after examining implications of blockchain in general for the profession, key questions and considerations are presented for practitioner consideration moving forward. Written with both practitioners and academics in mind, this research covers emerging topics of concern, and recommends possible steps toward resolving several outstanding questions in the blockchain space.
\end{abstract}

Keywords: Blockchain, Tokens, Tokenization, Accounting, Finance, Reporting

\section{Introduction}

Blockchain is perhaps and possibly without debate in some certain circles, the most dynamic and paradigm shifting technology to impact the financial services profession since the integration of the internet and personal computer into the business decision making process. Specifically, and even taking into account the false starts, failed pilots, and other obstacles that continue to arise in terms of widespread blockchain implementation, it does appear fair to state the following. Blockchain technology may have originated with the bitcoin blockchain, but that is far from the only iteration and version of blockchain that will have 
quantifiable business impacts on organizations both in the current business landscape as well as going forward. This research is applicable for both practitioners and academic professionals, seeks to analyze and quantify the impact of one specific development and application; tokenization. Prior to presenting this analysis, however, and attempting to project the effect of tokenization and other emerging applications on blockchain for business, it is important to revisit some of the effects blockchain is already having on the broader financial services marketplace.

The current market reality of the situation is that, even though large amounts of both financial and human capital have been invested in the broader blockchain ecosystem, there does appear to be large amounts of potential versus actual implementation in the marketplace. For any analysis and research of blockchain technology, or any emerging technology, to be accurately presented and discussed, a review of both current academic and practitioner literature should be conducted. Drilling down there appear to be a few definitive areas in which blockchain and blockchain applications are already having, and will continue to have, on how the different aspects of financial services interact with both other functional finance areas as well as other departments and expert groups. While no literature review is entirely comprehensive or exhaustive, this review is conducted both to level set awareness of the end users of this research as well as lay the foundation for an analysis of the next level application of tokenization.

\section{Literature Review}

Blockchain, when analyzed through an objective and impartial lens, does not in and of itself represent a particularly innovative or new technology tool, but rather a combination and integration of different technology platforms into one tool. The distributed and decentralized nature of the platform itself forms both the core of the concept, as well as representing a potential paradigm shift for financial services across the board (Carlozo, 2017). In essence, and without diving too much into the underlying computer programming and technology, this means that every computer (or node) that is linked up to the blockchain network has an up to date record of what exactly has transpired on the blockchain network. In addition to this information being communicated between different network members on a nearly continuous basis, the records of information themselves are permanent in nature. This immutability function is often interpreted or thought to mean that there is no way to edit, update, or change the information that has been uploaded onto a blockchain. While a simplistic view and interpretation of how this immutability function works, this represents an incomplete view of what immutability means from a practical perspective.

Each individual block of information is indeed an unalterable record, and is assigned a unique has identifier linked to the information included in the block itself. If at some point in the future a member of the blockchain wants to edit (for ethical reasons or not), this block will no longer sync with the previously assigned hash identifier and not be accepted by the members of the network. This linkage, connecting the individual blocks of data themselves with the other members of the network, points directly to another core component of how blockchain technology functions; consensus based verification (Carlozo, 2017). While the specific 
consensus based verification process will vary from blockchain to blockchain, the core underlying point of this idea is that in order for any new block of information to be added to the blockchain it must be approved by other members of the network. This consensus based methodology for adding the information to the blockchain itself helps ensure that unethical activities or actions may not be loaded onto the blockchain. While this is not a perfect solution it does assist in creating a self-reinforcing network where all members are equally as interested in maintaining the validity of the data itself. Since all network members receive up to date copies of the transactional data that has been uploaded onto the blockchain itself, and rely on this information to make business decisions, it is logical to conclude that members are interested in reinforcing the validity and accuracy of information.

For the purposes of this analysis, it is appropriate to set basic working definitions for blockchain terminology to both level set the expectations of the end users of this research, and to also ay any potential miscommunication that may have occurred as a result of the numerous definitions that have been created as a result of the variety of publications in this topical space. On top of the understanding of blockchain terminology itself, it is also important to recognize the reality that accounting is already merging and becoming integrated with other data based fields such as big data, data analytics, and business intelligence (Drew, 2018). In addition to the points below, Table 1 summarizes how blockchain is fundamentally different from existing technology and accounting systems, even those that operate on a cloud based format or via a decentralized hosting company.

Key traits and characteristics of blockchain to consider when analyzing blockchain options include the following:

- Decentralized and distributed in terms of accounting and financial information, represents a complete and fundamental shift from how the vast majority of accounting and financial information has traditionally been stored, processed, and handled. In virtually every other technology integration, upgrade, or development, the shift has been toward more of a centralized model. While that does indeed increase efficiency and consistency with regards to data processing, it also creates a single point of failure that can be both exploited by unethical actors as well as by natural disasters.

- Consensus based validation. Again, versus the traditional nature of financial information, in which a centralized authority - be it a financial institution, regulatory agency, or payments processor, has ultimate approval power over information as it is uploaded on to the system, blockchain protocols operate quite differently. While the specific protocols may vary, and often do vary, from blockchain to blockchain, the underlying assumption that each block of information must approved by a number of different, and independent actors, decreases the likelihood that any one unethical actor will be able to inject erroneous or fraudulent data into the ecosystem.

- Immutability. It is worth pointing out that the blockchain itself is not absolutely immutable, with future blocks certainly able to include adjusting information, the implications associated with the immutability of an individual block are difficult to overstate. If for example, there is a dispute as to which individual or institution uploaded certain pieces 


\section{MInstitute ${ }_{\text {Mnk }}^{\text {Macrothink }}$}

International Journal of Accounting and Financial Reporting

ISSN 2162-3082 2019, Vol. 9, No. 1

or blocks of data, it is entirely possible to go back, trace each block (which contains transactional information) and determine exactly when that block has verified and approved by the underlying consensus protocol.

Table 1. Comparison of blockchain versus traditional database options

\begin{tabular}{lll} 
Characteristic & Blockchain & Traditional Database \\
\hline Storage & Decentralized \& distributed & Centralized \\
\hline Verification & Consensus based & Single source of authority \\
\hline Edit ability & Depends & Yes \\
\hline
\end{tabular}

The implications and applications for blockchain on the accounting and broader financial services space have been well documented and analyzed in numerous articles, books, conferences, and other events since bitcoin itself burst into the mainstream consciousness. Although it is true that blockchain is not accounting system or platform,and was not developed to facilitate accounting record keeping, the development, interest, investment, and applications for blockchain in the accounting space has continued to increase. It is true that many of the applications that have entered into the marketplace to date have been connected to the larger organizations, the ripple effects of this research and development will invariably be felt further down the accounting supply chain in the near term. Specifically, there are three primary areas in which blockchain is positioned to not only have transformative effects on accounting work in the future, but actually is already doing so in the marketplace.

\subsection{Audit and Attestation}

Perhaps the most obvious use case for blockchain from a financial services perspective is to apply this technology tool to augment, improve, or otherwise modify current audit procedures, and this is because the audit and attestation process as it is currently constituted does not truly fulfill the needs of the marketplace (Raphael, 2017). Focused exclusively on the financial information and results achieved by the organization, the current attestation protocols and processes do not address the changing needs of the marketplace. Financial results and performance are, rather obviously, what attracts the majority of attention and focus of financial end users, but blockchain can also assist with providing assurance and attestation work in broader areas. The immutability of the information stored on the information, coupled with the near instantaneous nature of how this data is distributed to different network members means that auditors and other attestation professionals can begin addressing the emerging needs of the marketplace.

Drawing the bright line between blockchain, attestation, and emerging areas of importance can be summarized as follows. As blockchain based technology and platforms continue to become increasingly mainstream and integrated within business operations the accounting profession at large will, by necessity, change and evolve alongside the changing marketplace 
(Jun \& Vasarhelyi, 2017). Especially as the Internet of Things becomes increasingly mainstream, there will be ever increasingly amounts of information and data available for management professionals to analyze, report, and leverage for decision making purposes. Specifically, and leveraged with both automation, robotic process automation, and integrated artificial intelligence and other efficiency tools, this will enable auditor and attestation professionals to offer guidance and advice on a broader array of information sources.

\subsection{Reporting}

Reporting information and data to the marketplace represents one of the few fundamental traits and services that financial services professionals deliver to the marketplace. Blockchain, both in the form of current applications as well as projects currently underway in various stages, is already changing how data is reported. While it is true that there remains work to be done in terms of how scaling blockchain applications for commercial implementation and applications, several key differentiators and factors appear to be emerging.

First, since the information and data stored on the blockchain is updated as it is added to existing blocks, this data is distributed to network members on a continuous basis. Since most blockchain, as of this implementation, has tended to be focused on supply chain applications, this appears a logical place to analyze. Supply chains may initially bring to mind an image of physical goods or materials transported on a global basis, but the concept and idea of a supply chain indeed have much broader implications. Especially in an increasingly digitized environment, the idea of a supply chain can be interpreted as digital in nature. Different sources of information, depending on which organization is the focus of the current analysis, may be more or less important to different stakeholders or end users groups, but the underlying trend is consistent. Put simply, as increasing amounts of data - from healthcare to food safety to the underlying financial documentation underpinning global transactions becomes digitized, the expectations of stakeholders to have access to data will increase.

Blockchain, by the very nature of the concept itself, allows network members to see - in real time - the different types and sources of information generated by the organization and other network members. In addition, the consensus based way in which this data is added to the blockchain in the first place means that, in simple terms, bad information may be less likely to be uploaded in the first place. The greater supply of information and data, from any number of sources, presents a challenge for audit, attestation and reporting services that have traditionally only focused on financial information, further challenging the status quo for accounting and other financial professionals (Borthick \& Pennington, 2017). On top of this, the speed with which information is communicated between network members - nearly continuous in pace, allows the different members to update and monitor the flow of information throughout different members. Second, and building on the greater technology integration throughout the business landscape at large, speed and the speed with which organizations can leverage and analyze pieces of information can form a competitive advantage both in the current marketplace as well as going forward. 


\section{Tokenization}

While the implementation and different applications being constructed on various blockchain platforms are already driving innovation and disruption within the financial services landscape, the development of tokenization appears to represent an application merging physical assets with the digital blockchain environment, including those driven by cryptocurrencies (Diaz-Santiago \& Rodriguez-Henriquez, 2016). The idea of assigning tokens to represent physical assets or items in a digital environment is not, in and of itself, an innately new or innovative concept. In essence, what a tokenization process accomplishes is to disseminate access, ownership, or information across a wider array of potential owners or investors than would otherwise be possible in a strictly physical environment. Layering the idea of tokenization onto the already potentially disruptive nature of blockchain technology itself means that the process by which tokens can be issued can either be automated or operated in a completely decentralized nature. Technical specifications aside, it is important from a financial perspective to establish a working definition as to what tokenization represents as well as potential implications for the financial services sector. Table 2 summarizes and illustrates the connection between security and utility tokens, as of this writing, and additionally how these assets compare to equity securities.

Table 2. Accounting issues and differences between tokens and equities

\begin{tabular}{llll} 
Accounting Issue & Security Token & Utility Token & Equity Security \\
\hline Reporting & Equivalent to equities & Evolving & Established \\
\hline Taxation status & Evolving & Evolving & Established \\
\hline Asset classification & Equivalent to equities & Evolving & Established \\
\hline
\end{tabular}

For the purposes of this research a working and functioning definition of tokenization appears to be something along the following lines. Tokens may be described or analyzed in a broad sense in many conversations, but it is important to remember that token may originate from a variety of different sources (King, 2015). Tokens may, and often are, generated as a result of an ICO process in which an organization issues these tokens in an effort to raise capital. Drilling down specifically into this origination of tokens there are two primary categories within which tokens are classified in the U.S. under current guidance. Guided by the Howey test - a 1946 court decision - the S.E.C. has created two distinct categories of tokens that are generated a result of the ICO process. Security and utility tokens, although both originated as a result of an ICO and both categorized as tokens, are unique and distinct items are classified, reported, and accounted for different.

Security tokens are, in essence, the equivalent of issuing equity shares via an IPO process as far as the S.E.C. is concerned, and that is due to the following two considerations (Roberts, 2017). As per the Howey test if the investor is entitled to either a share of the profits of the organization, or can reasonably expect to exercise some level of management control of 
benefit from said investment, these tokens are classified as if they were equity securities. As per S.E.C. guidance and position papers, this is the category of tokens that is officially recognized, with the utility token category appearing to be much more difficult to achieve. Tokens that have been issued as part of an ICO process and been classified as security tokens, with additional complexity to the accounting situation, are classified and treated as property for income tax reporting purposes. Put simply, that means that for reporting, compliance, and documentation purposes there are multiple treatments that need to be considered (Aelbrecht, 2017).

In theory, utility tokens can be thought as equivalents to coupons or other means by which investors can access the goods and services of the issuing organization. While these tokens can be issued as part of the capital raising process, the tokens themselves do not grant the holders of said tokens any rights to entitle them to a share of organizational profits or any say in how the organization will be managed either presently or going forward. Instead, these utility tokens are meant to be redeemed by owners or investors in these utility tokens to access the goods and services of the organization instead of paying for them in cash. As seemingly logical as this classification may be from an operational perspective, the vast majority of instances of token issuances have been classified as security tokens. That said, and as noted in Table 2, much of the regulatory and accounting regulation surrounding these assets are still evolving due to both the nascent nature of the asset class itself and the dearth of instances in which utility tokens were actually recognized as legitimate.

\subsection{Accounting Related Tokenization Questions}

Regardless of the specific classification in question, whether it is security or utility token, there remain several unresolved questions and issues with regards to how these items should be accounted for and reported. The accounting implications and possibilities linked to these different classifications of tokens is a complex and fast moving area, but the following issues do appear to be core to the analysis.

First, are the proceeds generated from the sale of tokens a fundraising activity? If the answer to this question is yes, which may seem appropriate in the context of security tokens the following two sub-questions will be generated. First, are the proceeds taxed as regular income generated from operations? Second, and conversely, are the proceeds of a token issuance instead taxed as capital gains since the proceeds received a result of the issuance are used for equity and capital rising. On the opposite side of the aisle, if the proceeds linked to an ICO or other form of token issuance are not taxable, this raises the question of when these items are indeed going to be taxable?

Second, how is the basis determined for security or utility tokens? A key consideration of accounting and taxation is the establishing of an appropriate basis from which further calculation and reporting functions can be performed (Castano, 2018). That said, and even though basis does indeed form the foundation for both tax and financial reporting, this remains somewhat of an ambiguous issue with regards for basis issues. Drilling down specifically, but while remaining focused on the accounting and financial reporting aspect, the following issues can easily arise. If security tokens are indeed issued, and are issued for 
cash proceeds directly, does that form the basis for those tokens? Or, as might make more logical sense given the market based nature of cryptocurrencies, the basis only be established once a market for these tokens has been established? Linking back to a fundamental concept of the marketplace, assets of any kind only have value if they are valued by a third party. Returning to the issue of utility tokens, do these items have a basis for financial and tax reporting? Considering that in order to qualify as a utility token, the function of said token has to be more closely linked to that of a coupon or Groupon it would appear difficult to establish an externally verifiable basis for utility tokens given this data. For example, if the tokens are exchanged for the goods and services of the organization, does that form the basis for the tokens? Given the reality that as far as the IRS is concerned, any transactions of coins and tokens incurs a taxable event, linking the token to the value of the services may result in different tax impacts for different individuals.

Additionally, if the tokens are airdropped, are these tokens or coins treated or accounted for differently than tokens issued via an ICO process? Given the fact that a single airdrop, that associated with stablecoin Stellar, was able to raise \$125 million, this is not an idle question or matter that could be relegated to only cursory analysis (Roberts, 2018). From an operational perspective, an ICO almost always involves the exchange of financial resources or assets for tokens regardless of the nature of those underlying tokens. Contrasting with that, an Airdrop process may or may not require an exchange of value between different parties, but instead only involve action on the side of one of the parties. Drilling down, there appear to be two general categories in which Airdrops can fall within. Airdrops that require action on the part of the investors or the individuals or institutions actually sign up for, or obtain appear to more closely linked or connected to the issuance of a token as via an ICO or ICO-like process, since the investors did indeed have to take action to obtain custody of these tokens or assets. While definitive guidance, once again, has not yet been generated in U.S. markets, it would seem reasonable to expect such guidance to be generated by 2019 or 2020 . Airdrops, contrasting with the first example, that do not require action on the part of the token receivers, can generate additional questions that need to be considered. For example, if the actions of the organization that is conducting the airdrop are unknown to some of the end receivers, do those receivers truly have custody or possession of these assets? If they do indeed have custody, but the tokens - as is often case - have no true utility at the time of issuance, can a basis be reasonably determined? Lastly, and building on these other potential issues, what methodology is utilized to determine the basis of said tokens if that is indeed required.

Another interpretation of tokenization on the blockchain represents the decentralized and distributed ownership or investment in real world physical assets between various network members. An example of how such a scenario might actualize in the marketplace would be something like the following.

\subsection{Tokenization of Physical Assets}

An owner of a real world physical asset such as real estate is seeking to raise capital while not completely trading ownership in the asset for the capital that is raised. At first glance this may 
represent a crowdfunding or similar Kickstarter-like campaign, and there are similarities in how capital is raised from a wide array of potential investors without an exchange of true ownership. An additional definition and corollary has to do with the reality that virtually every blockchain model has issued a token that can be used to purchase or access services generated via that specific blockchain platform.

In terms of accounting implications of different tokens there appear to be several core questions and topics that need to be addressed and assessed before any definitive guidance will be able to issue by a professional or legal body. Different classes of tokens may very well result in different accounting treatment and standards, but there are core issues that should form the basis of an analysis, debate, and conversation in the broader tokenization space.

\subsubsection{Accounting Considerations for Tokenization Accounting}

- How are these tokens generated? Linking back to earlier analysis on the various methods by which tokens can, and are, being generate their purpose and end user and entered into the marketplace, this will make a difference as to how these specific tokens are treated. Core issues that appear to be relevant to obtaining an accurate answer to this question include the following core areas. First, are the investors or owners of these tokens providing compensation to the token creator? Second, and a related question in this same topical area is what entity or institution is actually generating and distributing said tokens. Is the owner or the institutions that owns the underlying asset, or is it a third party intermediary that is taking responsibility for the issuance of these tokens?

a. Each of those scenarios will generate different liabilities, obligations, and rights for the parties that are involved in the transactions themselves, especially due to the lack of definitive and authoritative guidance in the space.

- Are the tokens themselves then traded on an exchange, or simply held in a custodial-like account? In tokens become actively traded, this increases the liquidity and potential value of these tokens, but also lead the way to a value being established for basis, tax reporting, and financial reporting purposes. Depending on how widely traded these underlying tokens becomes; they may indeed fall under the classification of security tokens as established by the SEC and become governed by those security token rules. Conversely, if the tokens generated to represent ownership of the asset do not become actively traded, and instead as used to gain access or use rights to the underlying asset itself, then these might very well form the foundation for classification as a utility token.

b. For tokens, even those classified as utility tokens due to, have a value assigned to them as a result of the access they grant in terms of using the underlying asset itself that may very lead to a value and market cap being assigned to said tokens. In any case, financial practitioners need to be aware of both the uses of the tokens generated via this process, and any valuation methodologies that could reasonably be used to establish a basis for future comparison.

- Governance issues must be brought to the forefront when considering the issuance and accounting for these different cryptocurrencies and cryptoassets. In virtually every cash and 
associated with every cryptocurrency or cryptoassets there is a certain level of anonymity associated with the different tokens and cryptocurrencies. Given this lack of transparency on one hand, and the ability of other network members to examine and review the blocks of data on the chain, this represents a unique dynamic challenge for financial practitioners.

c. During the issuance process of different tokens and coins, for example, there should ideally be some sort of identification as to which organizations or individuals are actually receiving the funding itself. Even if after the issuance there is a level of anonymity associated with the cryptocurrencies themselves, establishing a proper audit and attestation trail to link together ownership and fundraising appears to be a logical step to help encourage more widespread adoption for business use.

d. If there are instances when the issuing company associated with the token or coin goes bankrupt, otherwise fails, or is experiencing financial instability or difficulty, how are responsibilities ultimately assigned to provide redress to stakeholders and investors? In other words, are the investors that have put capital to work in a token or coin provided with contact information or the ability to seek redress or the ability to made whole in the event of a business reversal?

- What are the assurances protocols put in place to verify, existence, and ownership of these tokens, as well as what specific rights they deliver? Auditing potentially anonymous data and information is a challenge even under the best of circumstances, but can become even more complicated when the conversation move outside of the established cryptocurrency conversation linked to bitcoin and toward emerging areas such as ICOs and other tokenization processes. Drilling down from an accounting perspective, it would be logical to conclude that several of the following topics will increasingly emerge as important and core to just how cryptoassets and tokens. Building on the existing framework surrounding audit and attestation professionals, namely the existence and valuation of different kinds of assets, an appropriate framework will include the following.

\section{Assurance and Attestation Considerations for Cryptocurrency Tokens}

The proposed framework herein is not meant to represent authoritative guidance not definitive recommendations for the accounting and reporting of different cryptoassets or other items. Rather, these items and talking points should be thought of a list of suggested best practices of items to consider and think about when financial services professionals are weighing different options as to how to account for and repot different classes of token or tokenized assets.

First, legal experts and advisors should form a core part of any team or group tasked with the proper assessment, reporting, or analysis of different tokenized assets, and not just because of the current uncertainty with regards to this area. In addition to the uncertainty currently in the space, it is also important to have individuals on the team that have expertise with regards to securities laws, tax implications of different equity and financial issuances, and a familiarity with navigating the legal ins and outs of different capital raising initiatives (Moynihan \& Syracuse, 2018). As of this writing the S.E.C. C.F.T.C., and various state agencies are 
weighing in with regards to just how these different tokens and assets should be reported, examined, and communicated to the marketplace. Although some guidance certainty does exist, such as the differentiation between security and utility tokens, definitive and authoritative accounting guidance remains a work in progress (Walch, 2018).

Second, and building on the reality that definitive accounting guidance has yet to be issued, any reporting or guidance offered in response to cryptoassets or token reporting should be issued with a caveat connected to the changing nature of accounting guidance. In other words, the current opinions and recommendations issued by financial practitioners will, almost invariably, not be entirely in compliance with the end resulting regulation eventually issued. The takeaway for practitioners is that, even when consulting with clients and offering guidance in response to specific questions, the reality of this uncertainty must also be a part of the conversation. For example, and perhaps an obvious but often overlooked step, is to certainly preserve and maintain copies of records, documentation, and other information that has driven the decision making process. Such steps should already be in place regardless, but is an especially important step when considering the emerging nature of cryptoasset and token reporting.

Third, education and training both on the side of practitioners and clients themselves must form a core component of the services and guidance provided by financial professionals. While it is may seem clear that financial practitioners will need to update knowledge and skills linked with regards to this emerging area the education of clients and customers must also be part of the services provided by financial services professionals. Especially as many clients might be interested in investing in tokens and other cryptocurrency assets the lack of robust and comprehensive materials in the space provides an obvious vector for unethical activity to occur. In terms of education and training, it appears reasonable and logical to conclude that the training and education itself should be comprised of two distinct components. First, basic education in term of terminology and conceptual knowledge needs to be addressed and explain both to

Accounting classification and guidance is an essential next step that must be completed to help facilitate the broader adoption and utilization of cryptocurrencies are currencies and align them more with mainstream usage. In addition to assisting with the broader usage of said cryptocurrencies and cryptoassets, encouraging investors and end users to use them not only as an investment but rather as a viable currency tool. As of current market literature and evidence, there does not seem consensus around just how to measure, classify and report these different cryptocurrencies, which has also led to some uncertainty in the space. Specifically the emerging perspective that appears to be holding true is that many practitioners seem to be coming around to the point of view that cryptocurrencies should be treated and reported as intangible assets. If this perspective, which is non-authoritative in nature, does indeed become the mainstream opinion and way of reporting cryptocurrencies, there are several issues that must be addressed along the way.

For the purposes of accounting and reporting, how is the fair value of these different assets determined, i.e. if market price is utilized where do these market prices which marketplace or 
exchange is the standard setting body? In the case that cryptocurrencies and cryptoassets are treated as intangible assets, how often are these assets tested for impairment? Following that is this treatment and classification actually correct in the terms of how reflecting market realities. Even if the technically the treatment may be accurate and correct, it may not actually reflect the way in which the technology works. With these various options, questions, and points of contention that have been raised in the context of how to perform the accounting for tokens and cryptocurrencies, it also appears logical to include some thoughts and comments as to how the accounting and financial services profession will evolve and change moving forward. It is reasonably clear that the broader business landscape that financial services practitioners must operate within is continuing to change at an accelerating pace, and that practitioners must also develop skills and competencies to navigate the marketplace. While not presented as an all inclusive or exhaustive list of points and factors to consider, these suggestions and points of focus should assist practitioners to both develop and maintain new competencies linked to emerging market forces.

\section{Future Directions}

As of this research, the field of tokenization, accounting for various tokens and cryptocurrencies, and the developing of standards and frameworks still appears to be an emerging area of professional importance. Acknowledging this reality, it is difficult to project with a significant level of confidence as to what exactly will be the end state in terms of regulation of definitive accounting guidance, particularly on an international basis. While developing authoritative guidance and standards, as of this point, remains a work in progress, there are several traits and ideas that can be issued and stated as necessary for the profession moving forward. In order to develop into the strategic business partner or business advisor that is the oft-cited goal of practitioners, it will be necessary for the accounting and financial professionals to have a working understanding of emerging technologies and the impact these tools may have on the profession. Put another way, practitioners will - reasonably - be expected to assume a more strategic role more akin to advisory expert rather than focusing on traditional compliance oriented tasks.

Education, both on the part of practitioners and in conversations with clients, also forms a core idea that will be increasingly form a role that will have to be addressed and fulfilled by practitioners. On top of the rather obvious requirements for practitioners to remain up to date and well informed with regards to these emerging trends, conversations with clients and customers are also necessary. In addition to seeking financial advice and insights from practitioners, it appears logical to conclude that advice will also be sought with regards to the business applications of blockchain, cryptocurrency, and other emerging technologies. While this, the role of educator, may seem to be differentiated from traditional responsibilities of practitioners, this represents an opportunity to increase current service offerings as well as expand current lines. In any situation, the future success of a profession is dependent on expanding and developing new service lines and opportunities to keep pace and address the needs and expectations.

Asset tokenization and the broader introduction of financial tokens to the financial services 
landscape, certainly seems positioned to pose a challenge as well as present an opportunity for practitioners and researchers. These opportunities and challenges are not, however, linked solely to the idea of cryptocurrencies and tokens themselves, but rather are connected to a broader professional shift. Whether it is connected to the increased levels of technically based competition from technology tools themselves, it is increasingly apparent that the profession will be unable to survive and compete effectively using traditional skill sets and technique. Rather, the changes and implications driven and derived from tokenization and the broader cryptocurrency landscape are merely symptoms of a broader change already underway. Regardless of industry affiliation, however, it is increasingly clear that the professional landscape will change, and that professional's will the interest and ability to change will need to do so in order to succeed. Cryptocurrencies, tokens, and other cryptocurrency related assets will have an impact and effect on virtually every aspect of the professional landscape, but if practitioners and professionals are able to adapt to this change, these same forces will also generate new opportunities and career paths. While in the early stages, these technologies and affiliated applications appear posed to dramatically change the profession, work performed by practitioners, and the way in which practitioners engage with the marketplace at large.

\section{References}

Aelbrecht, J. (2017). SEC rules that digital tokens are securities. Global Investor, 1.

Borthick, A. F., \& Pennington, R. R. (2017). When data become ubiquitous, what becomes of accounting and assurance?. Journal of Information Systems, 31(3), 1-4.

Carlozo, L. (2017). Understanding blockchain. Journal of Accountancy, 224(2), 1.

Carlozo, L. (2017). What is blockchain?. Journal of Accountancy, 224(1), 1-2.

Castano, I. (2018). Cryptocurrency taxes to soar amid tighter rules. International Tax Review, N.PAG.

Dai, J., \& Vasarhelyi, M. A. (2017). Toward blockchain-based accounting and assurance. Journal of Information Systems, 31(3), 5-21.

Díaz-Santiago, S., Rodríguez-Henríquez, L., \& Chakraborty, D. (2016). A cryptographic study of tokenization systems. International Journal of Information Security, 15(4), 413-432.

Drew, J. (2018). Merging accounting with "big data" science. Journal of Accountancy, 226(1), 47-52.

King, W. B. (2015). Tokenization is still king, but will bitcoin and blockchain eventually take hold?. Credit Union Journal, 19(20), 15.

Moynihan, M., \& Syracuse, D. (2018). Blockchain, tokens, and mutual funds--we're not there yet. Investment Lawyer, 25(7), 11-20.

Raphael, J. (2017). Rethinking the audit. Journal of Accountancy, 223(4), 29-32.

Roberts, J. J. (2017). SEC says digital tokens are securities, warns of fraud. Fortune.Com, 105. 


\section{Macrothink}

International Journal of Accounting and Financial Reporting ISSN 2162-3082 2019, Vol. 9, No. 1

Roberts, J. J. (2018). Blockchain announces \$125 million "Airdrop" of Stellar Cryptocurrency. Fortune.Com, N.PAG.

Walch, A. (2017). Blockchain's treacherous vocabulary: One more challenge for regulators. Journal of Internet Law, 21(2), 1-16.

\section{Copyright Disclaimer}

Copyright for this article is retained by the author(s), with first publication rights granted to the journal.

This is an open-access article distributed under the terms and conditions of the Creative Commons Attribution license (http://creativecommons.org/licenses/by/4.0/) 